\title{
Long-term neurodevelopmental outcomes of infants born late preterm: a systematic review
}

\author{
This article was published in the following Dove Press journal: \\ Research and Reports in Neonatology \\ 9 November 2015 \\ Number of times this article has been viewed
}

\author{
Tanya Tripathi' \\ Stacey C Dusing 2,3 \\ 'Rehabilitation and Movement Science \\ Program, Department of Physical \\ Therapy, ${ }^{2}$ Department of Physical \\ Therapy, ${ }^{3}$ Department of Pediatrics, \\ Children's Hospital of Richmond, \\ Virginia Commonwealth University, \\ Richmond,VA, USA
}

Purpose: Late preterm (LPT) births constitute a large proportion of the preterm births in the USA. Over the last few decades, there has been an increase in research focusing on the neurodevelopment of infants born LPT. The purpose of this research was to systematically review the long-term neurodevelopmental outcomes in LPT infants.

Materials and methods: We identified studies by using PubMed, ERIC, CINAHL, and PsycINFO databases. The references of included papers were reviewed for additional papers that met the inclusion criteria. Included papers compared motor, cognitive, language development, or academic performance outcomes between individuals born LPT and a term control group assessed between 12 months and 18 years of age. The Preferred Reporting Items for Systematic Reviews and Meta-Analyses standards for systematic reviews were utilized including a two-step, two-investigator review process.

Results: Of the 4,720 studies found in the initial search, 20 studies met the inclusion criteria. Approximately $75 \%$ of the 16 studies that assessed cognitive outcomes reported cognitive delay in the LPT group when compared to their full-term counterparts. More than $50 \%$ of the seven studies that assessed motor outcomes suggested a delay in motor development in the LPT group in comparison to full-term. Fewer papers assessed academic performance and language in children born LPT; however, the majority identified borderline differences when LPT infants were compared to those born full-term.

Conclusion: Evidence suggests that infants born LPT are at an increased risk of neurodevelopmental delay between 1 and 18 years of life when compared to those born at term. The delay is most evident in the cognitive domain of neurodevelopment. Children born LPT are also at a risk of delayed language development, motor development, and lower academic performance. The rate of developmental delay is reduced somewhat when controlling for social factors; however, group differences persist.

Keywords: late preterm infants, developmental outcomes, motor development, cognitive development, language development and school performance

\section{Introduction}

A preterm infant, as defined by the American Academy of Pediatrics and the American College of Obstetricians, is an infant born before the end of the 37 th week (259th day) of pregnancy, counting from the first day of the last menstrual period. ${ }^{1}$ Infants born prior to 37 weeks of gestation are often physiologically immature and show restricted compensatory responses to the extrauterine environment when compared to infants born full term. ${ }^{1}$ Until 2005, infants born between 34 and 36 weeks of gestation were frequently referred to as "near term", giving a false impression of being similar to term infants in their risk of developing medical complications. ${ }^{2}$ Near term infants were
Correspondence: Stacey C Dusing Department of Physical Therapy, Virginia Commonwealth University PO BOX 908224, Richmond, AV 23298, USA

Tel + I 8048280234

Fax + I 8048288 III I

Email scdusing@vcu.edu 
treated by parents, caregivers, and health care providers as being developmentally equivalent to their full term counterparts. The lack of understanding regarding developmental biology was reflected in an increased rate of elective delivery during the last month of gestation. ${ }^{3}$ The National Institute of Child Health and Human Development (NICHD) held a workshop in which invited experts discussed the rising rate of preterm births, $70 \%$ of which was attributed to births between 34-37 weeks of gestation and the accompanying medical concerns. ${ }^{2,4}$ Following the Optimizing Care and Outcome of the Near-Term Pregnancy and the Near-Term Newborn Infant workshop, ${ }^{1}$ a recommendation was made to change the term near term to "late preterm" (LPT) in order to underscore the importance of regular screening of neurodevelopmental delay in this population. LPT infants are now defined as infants born between 34 weeks 0 days and 36 weeks 6 days of gestation.

LPT birth on its own is identified as an independent risk factor for neonatal mortality and morbidity. ${ }^{5}$ The mortality rate for infants born LPT is three fold higher than for those infants born at term. ${ }^{4}$ Infants born LPT are at an increased risk of respiratory distress, bronchopulmonary dysplasia, apnea, patent ductus arteriosus, feeding problems leading to compromised nutrition, hypoglycemia, hyperbilirubinemia, and hypothermia. ${ }^{6}$ LPT births are thought to have a major impact on the cost of health care and are identified as an important factor contributing to an increase in the rate of hospital readmissions, thus making them a matter of major health concern. ${ }^{4}$

The nervous system in an embryo matures a great deal during the third trimester of pregnancy, including a three- to fourfold increase in brain volume in the form of increase in gyri, sulci, synapses, dendrites, axons, oligodendrocytes, astrocytes, and microglia. ${ }^{1}$ Infants born preterm have an immature central nervous system with fewer gyri and sulci and a brain size of approximately two-thirds to the size of term infant's brain. ${ }^{3}$ This central nervous system immaturity places infants born LPT at risk of neurodevelopmental disabilities.

Over the last decade, many researchers have broadened their focus from determining the neurodevelopmental outcomes in extremely premature infants (23-28 weeks of gestation) to include outcomes of LPT infants. A 2011 systematic review of early childhood development outcomes of LPT infants identified ten papers published prior to March 2010. ${ }^{7}$ Their findings suggests that children born LPT are at an increased risk of adverse neurodevelopmental outcomes when compared to their full term counterparts between 1 and 7 years of age. While this review paper provided current evidence at its time, the purpose of our systematic review is to systematically review the long-term neurodevelopmental outcomes of infants born LPT, including the vast amount of new evidence published since the McGowan review in $2011^{7}$ and including literature addressing developmental outcomes in older children who were born LPT.

\section{Materials and methods}

\section{Search strategy}

We identified studies by using MEDLINE ${ }^{\circledR}$, Cumulative Index to Nursing and Allied Health Literature (CINAHL), Education Resource Information System (ERIC), and PsycINFO databases, and the reference lists of the included papers. We identified literature published from January 1990 to January 2015. The comprehensive search was undertaken using following search terms: Late preterm infants; developmental outcomes; motor development; cognitive development; language development and school performance. The Supplementary materials section provides a detailed list of search terms and Medical Subject Headings (MeSH) used for each database.

\section{Selection of eligible studies}

The preferred reporting Items for systematic reviews and meta-analyses (PRISMA) standards for systematic reviews were utilized, including a two-step, two-investigator review process. Included studies compared motor, cognitive, language development, or school/academic performance between individuals born LPT and a term control group assessed between 12 months and 18 years of age. Studies were excluded if they met any of the following criteria: not published in English, no LPT infants group, studies not reporting a developmental outcome or not including a developmental assessment between 12 months of age and 18 years of age, and studies not comparing an LPT group with a full term control group. Studies that combined moderate/LPT subjects in one group were included if they examined a large cohort and the mean gestational age fell into the LPT range. During the screening step, the titles and abstracts of all papers identified in the search were reviewed and excluded only if they clearly did not meet the inclusion criteria. During the eligibility determination step, two reviewers used the inclusion and exclusion criteria to review the full text of all papers not eliminated in the screening step. Papers that met the inclusion criteria were included, and their references were reviewed to determine if any relevant papers were missed in the search.

\section{Data extraction}

For this study, we designed data extraction forms that included information regarding author name and location, 
objective/hypothesis, study sample and sample size, type of study, developmental domain assessed, and age of assessment, along with results of each domain assessed. These data were compiled for review and interpretation. Any disagreement between reviewers was resolved with discussion. A meta-analysis was not performed, given the wide variety of ages and outcomes included in the review.

\section{Study quality assessment}

The methodologic quality of the included studies was assessed by using the Critical Appraisal Skills Program (CASP) checklist for cohort studies. Only section A from the checklist was used for screening the quality of the study design (not analysis) of the studies included. Section A of the CASP checklist consists of eight questions, answers to which can range from "yes", "no", and "not clear", where "yes" is given a value of 1 and "no" and "not clear" are given a value of 0 . For this study, the research team considered cohorts to be recruited in an acceptable way if they included all infants born in a hospital or a random selection of infants, and studies appeared to have limited selection bias. LPT birth needed to be documented in the medical records vs parental recall to be scored as having minimal bias. Outcome measures needed to be completed by reliable and blinded assessors to be considered unbiased. Subject follow-up of $\geq 30 \%$ was considered acceptable. Using these criteria and the CASP guidelines, each study received a score in the range of $0-8$ points. ${ }^{8}$

\section{Results}

\section{Included studies}

The initial database searches retrieved 6,269 studies of which 1,549 were duplicates (Figure 1). Of 4,720 studies found in the initial search, 4,382 were excluded during the screening phase based on title and abstract review. Of 338 studies extracted during eligibility determination, 20 studies met the inclusion criteria (Table 1). A major proportion of the studies were excluded following full text review because the sample did not include an LPT group that was distinguished from other preterm groups, a full term control group was not included, results did not compare full term and LPT groups, or developmental assessments were completed prior to 12 months of age.

\section{Description of included studies}

Of the 20 included studies, ten studies focused solely on the LPT group and three studies included a subgroup of complicated LPT Infants who were identified with a history of neonatal intensive care unit (NICU) admission due to clinical instability

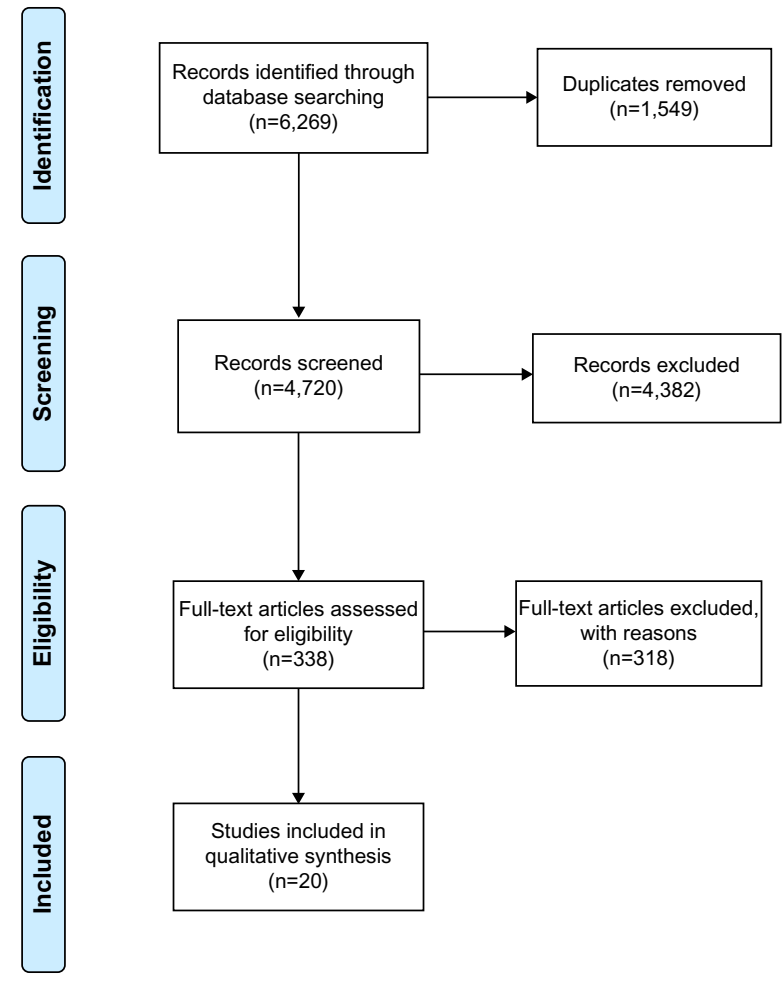

Figure I Flowchart of study selection process.

Abbreviation: n, number of studies included in the current review.

and/or birth weight $<2 \mathrm{~kg}$. All of the studies had been undertaken in past 13 years. Most studies were conducted in USA (ten studies), ${ }^{9-18}$ followed by the UK (four studies), ${ }^{19-22}$ Australia (two studies), ${ }^{23,24}$ Italy (one study), ${ }^{25}$ Spain (one study), ${ }^{26}$ Israel (one study), ${ }^{27}$ and Canada (one study), ${ }^{28}$ indicating a large proportion of research had been carried out in developed nations. Six of the 21 studies had a retrospective cohort design. Seven studies included analysis of national datasets, three studies were part of the Early Childhood Longitudinal Survey, Birth cohort (ECLS-B), ${ }^{11,12,18}$ two studies were part of the Avon Longitudinal Study of Parents and Children (ALSPAC) study group ${ }^{20,21}$ one study was the Cohort Millennium Study, ${ }^{22}$ and one study was the National Longitudinal Survey of Children and Youth (NLSYC). ${ }^{28}$ The majority of papers measured outcomes at age 8 years or less. No studies assessed outcomes beyond 15 years of age (Table 1).

The Cognitive Studies, Motor Development Studies, Language Study, School/Academic performance studies section of the result provide an concise review of the findings while Table 2 provides details of the findings in of each study. The study results are described under the four key long-term neurodevelopmental outcomes measured: cognitive, motor, language, and school/academic performance. Many studies included outcomes in more than one of the four domains. 


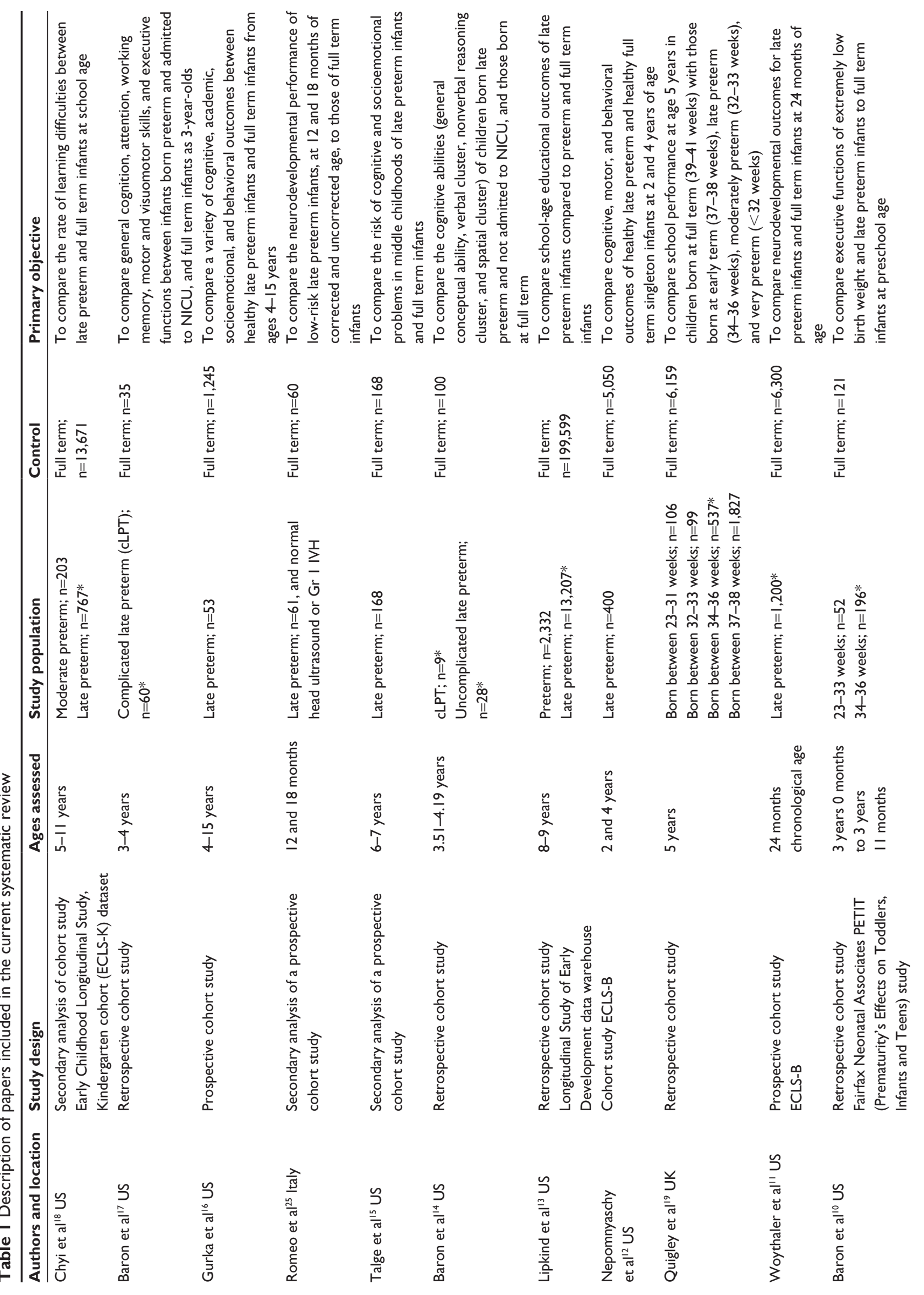



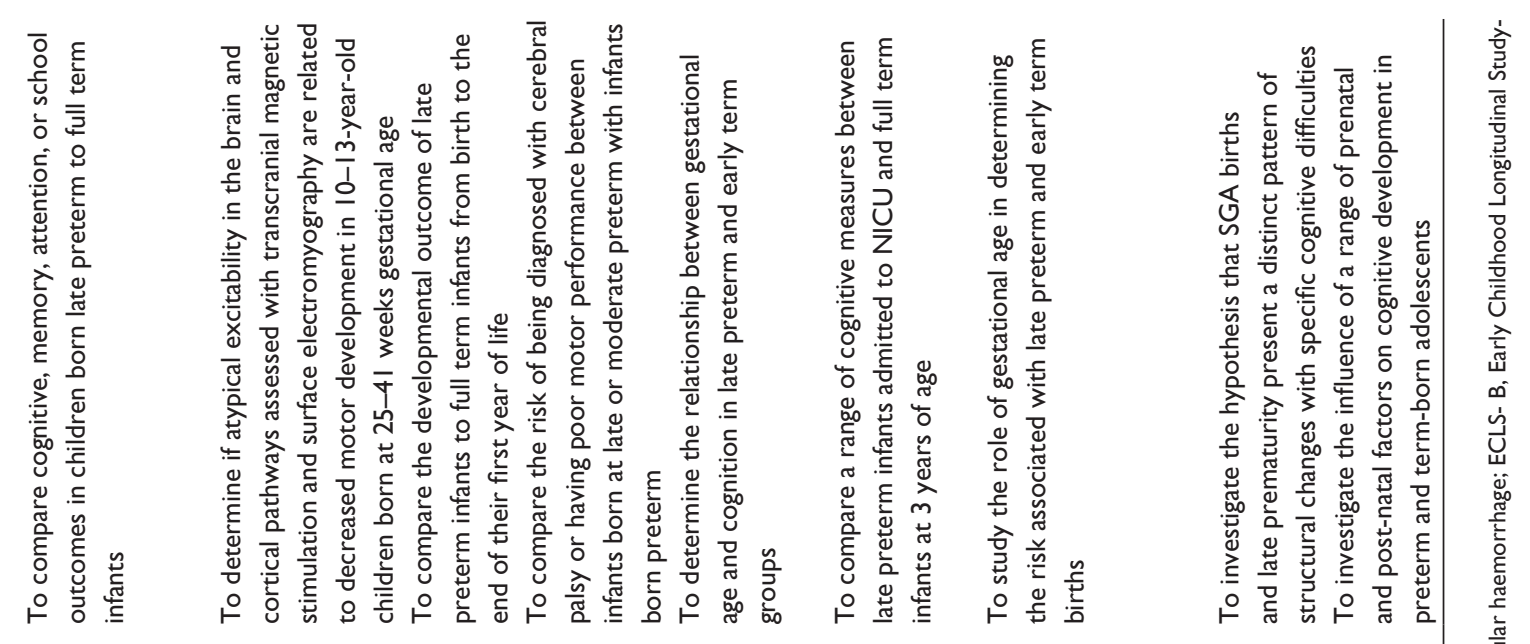

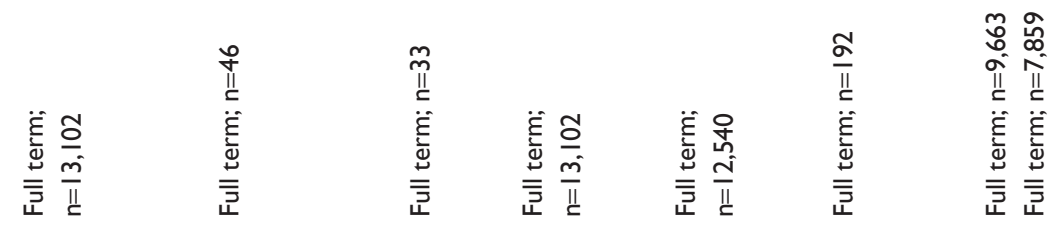
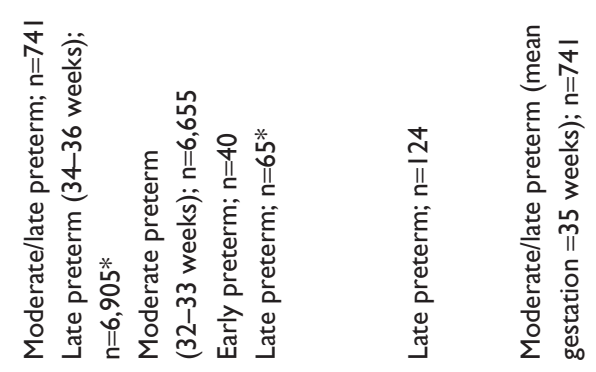

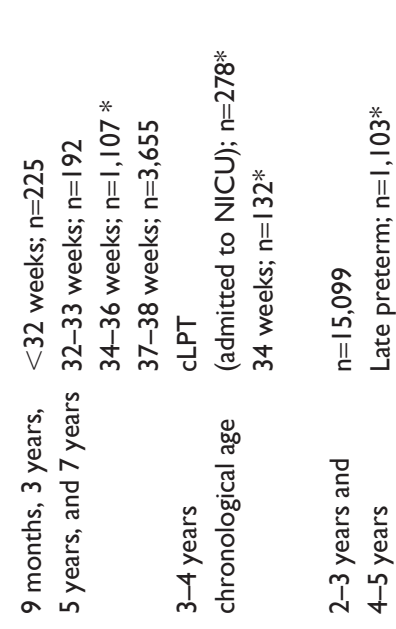
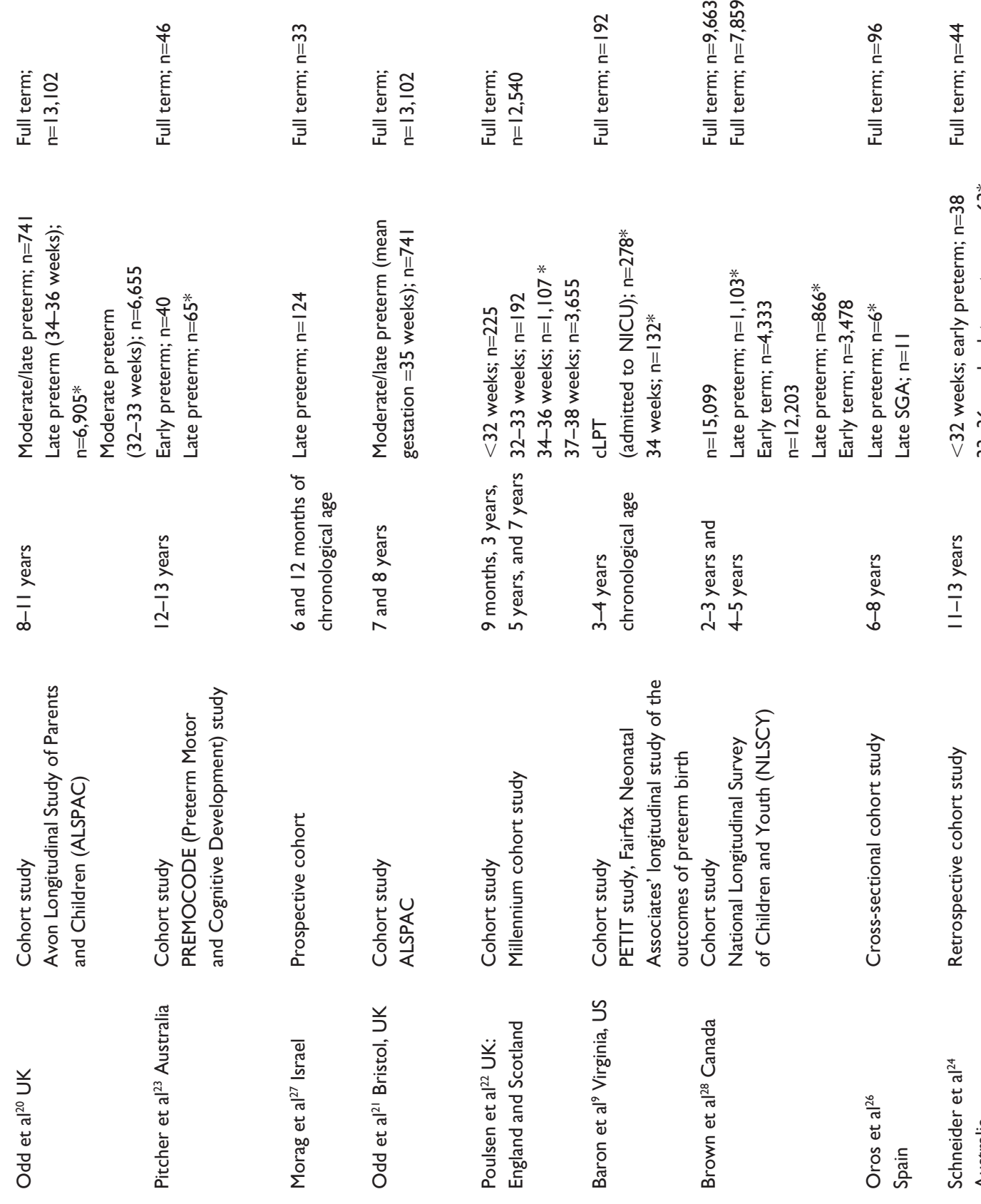


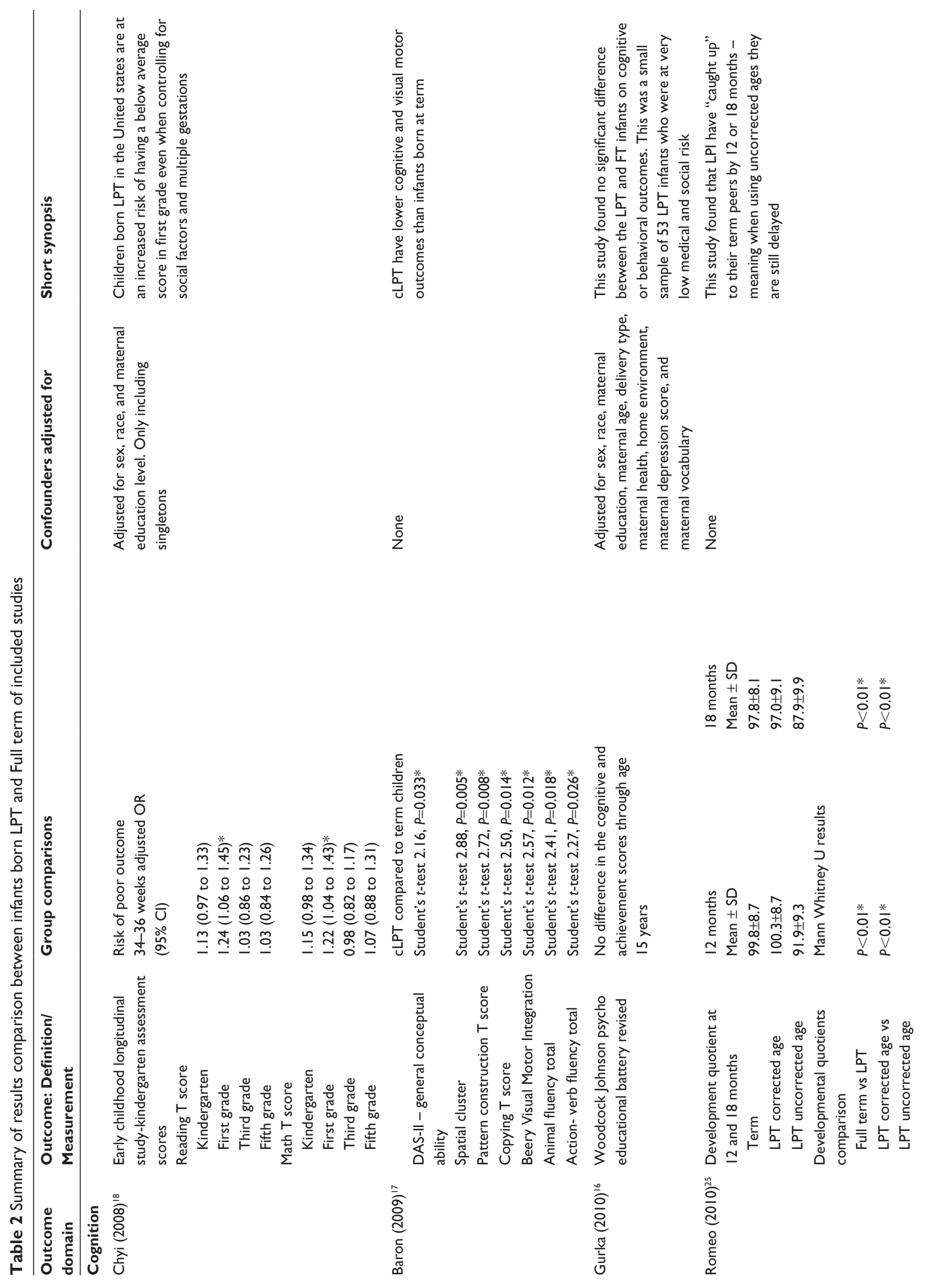



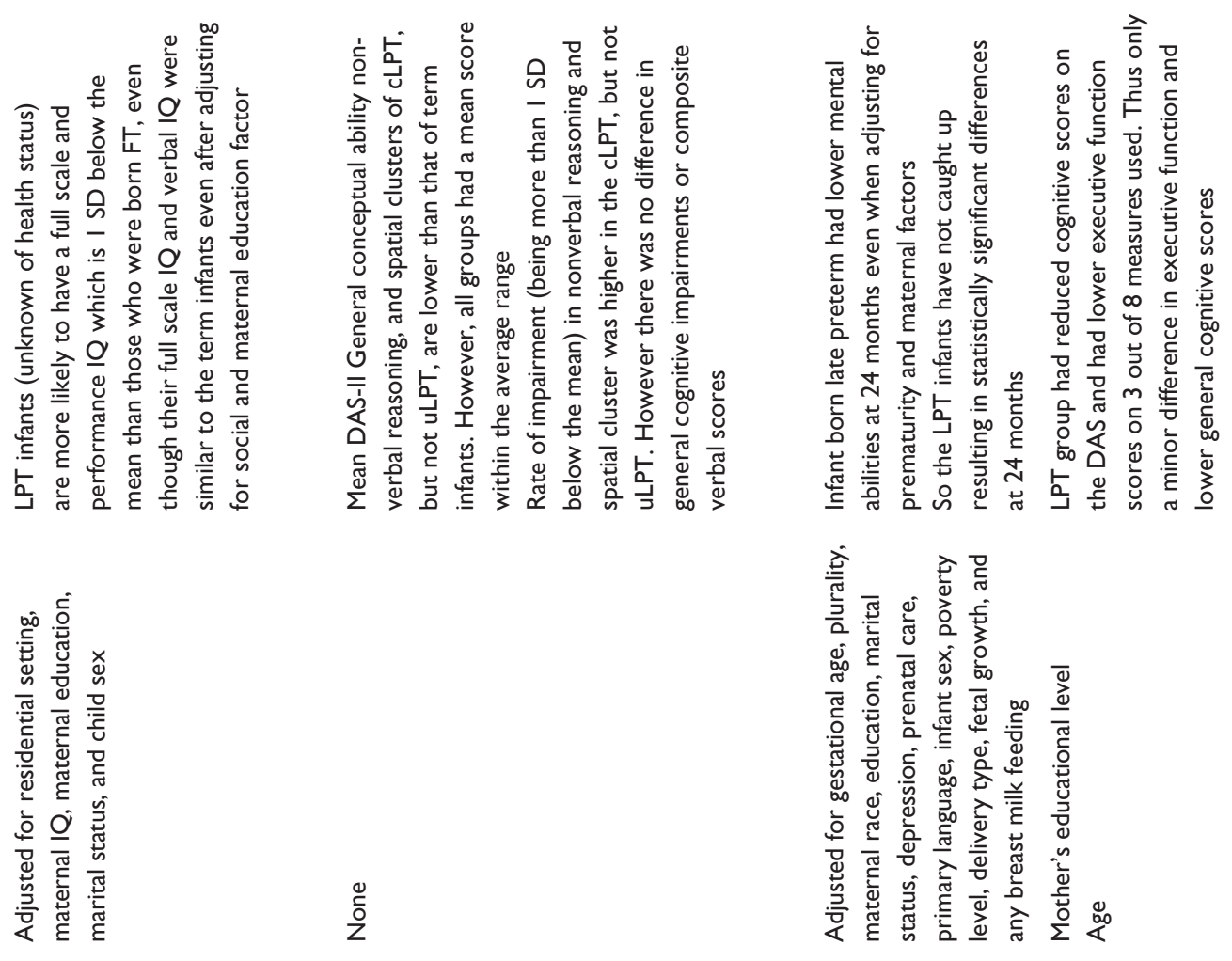

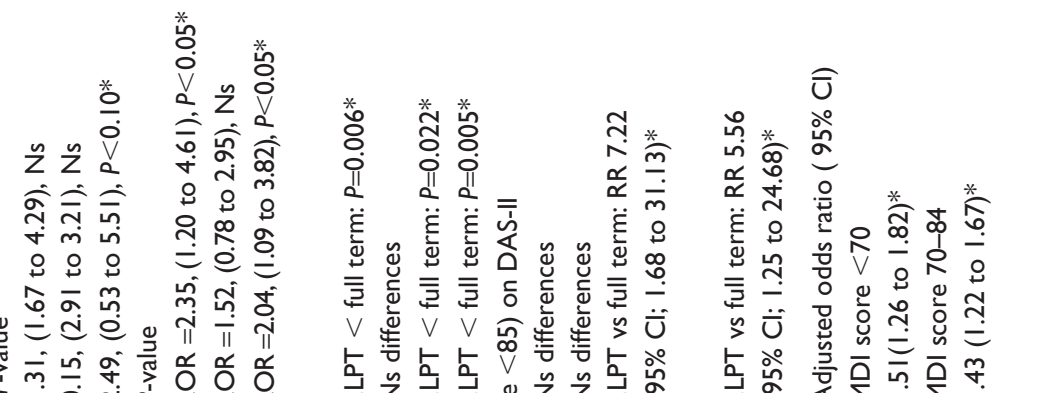

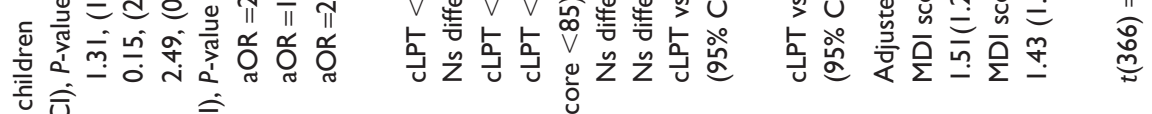

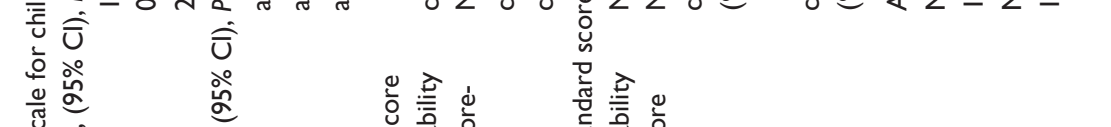

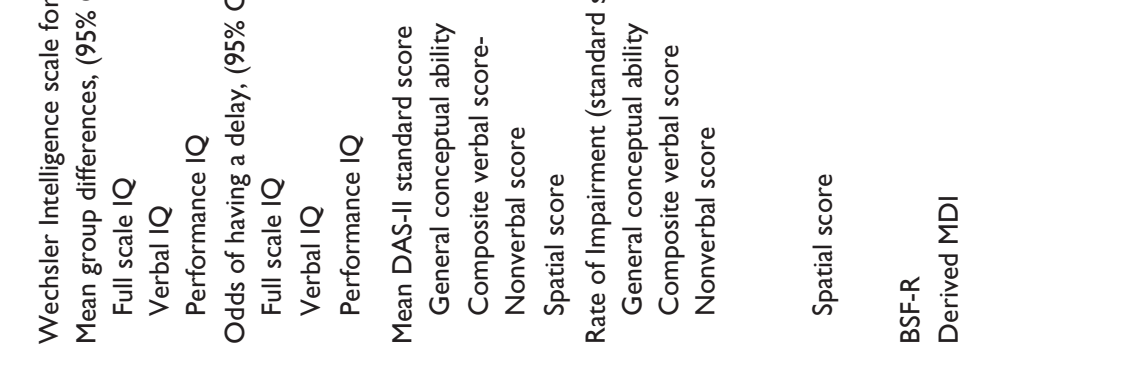

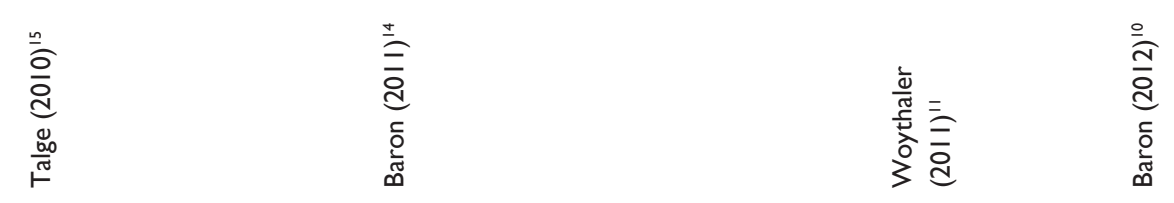

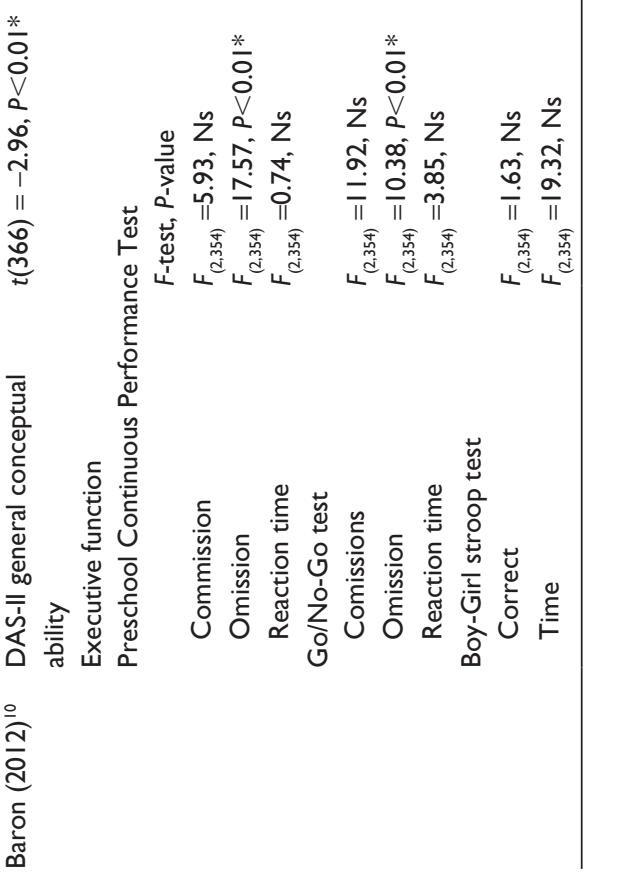




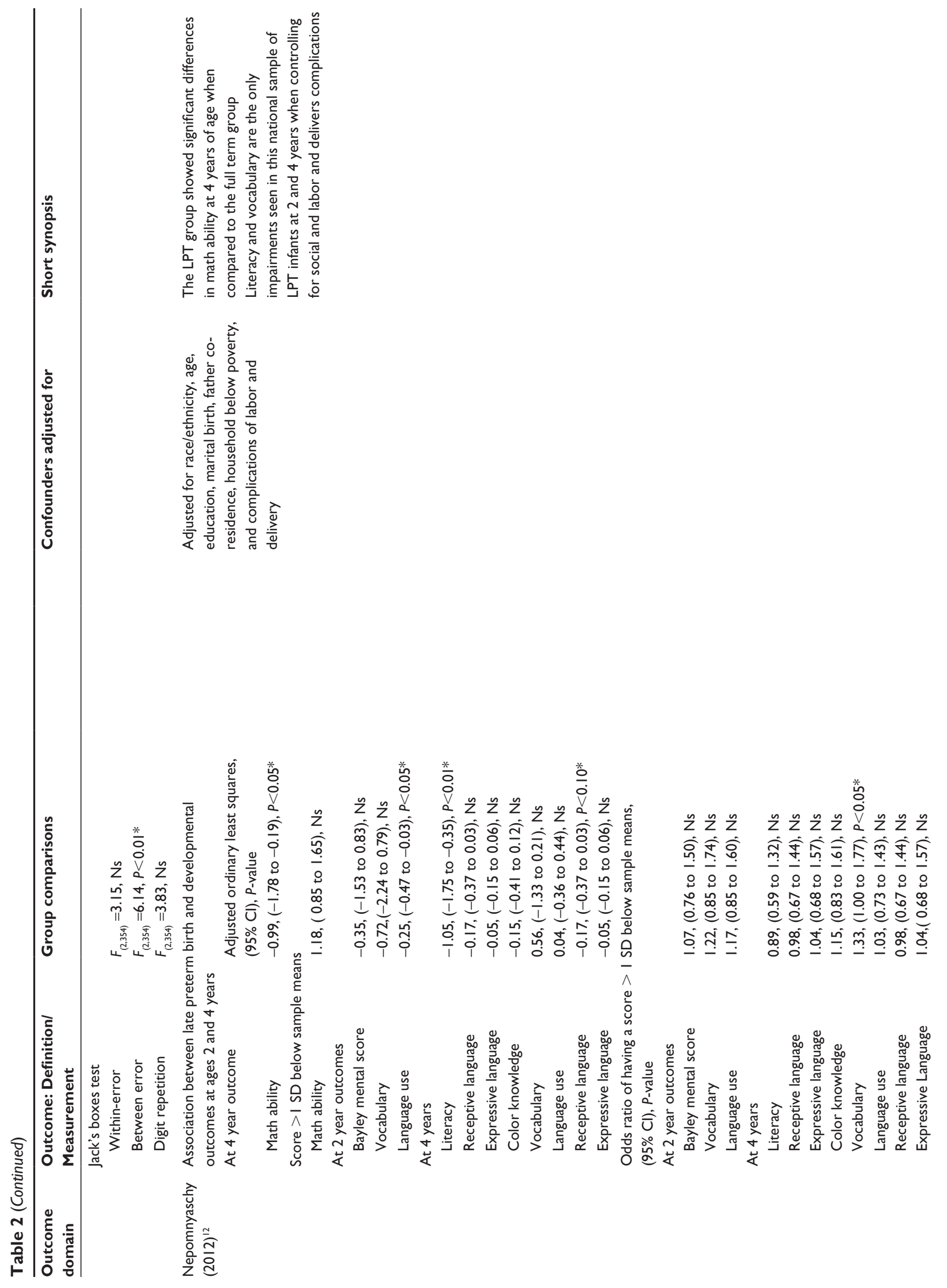



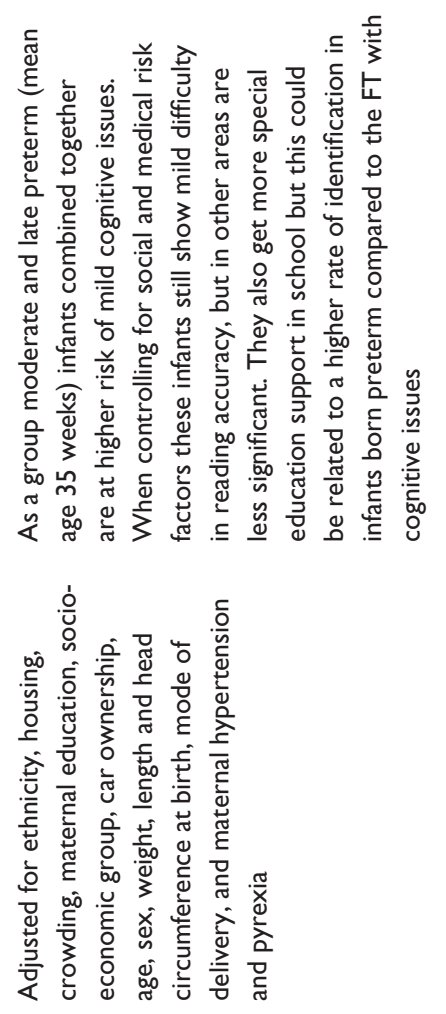

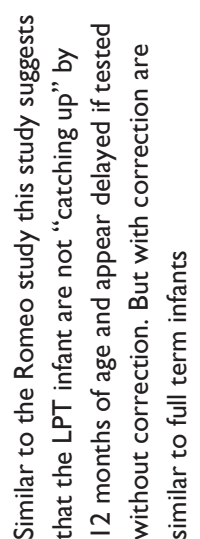

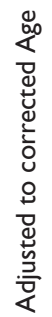

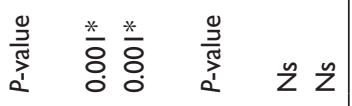

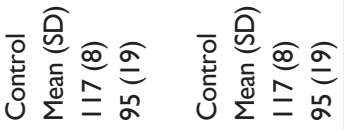

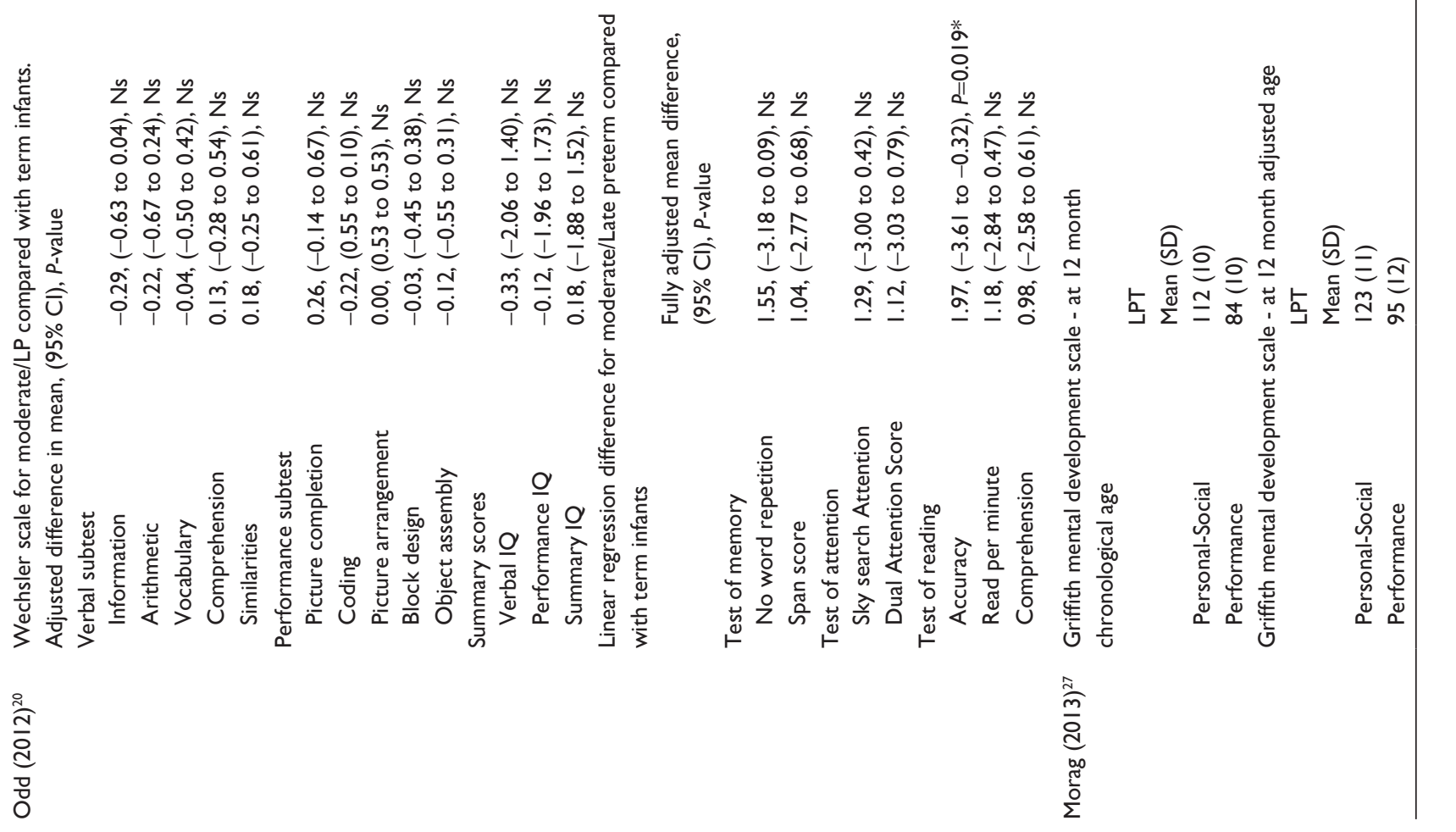




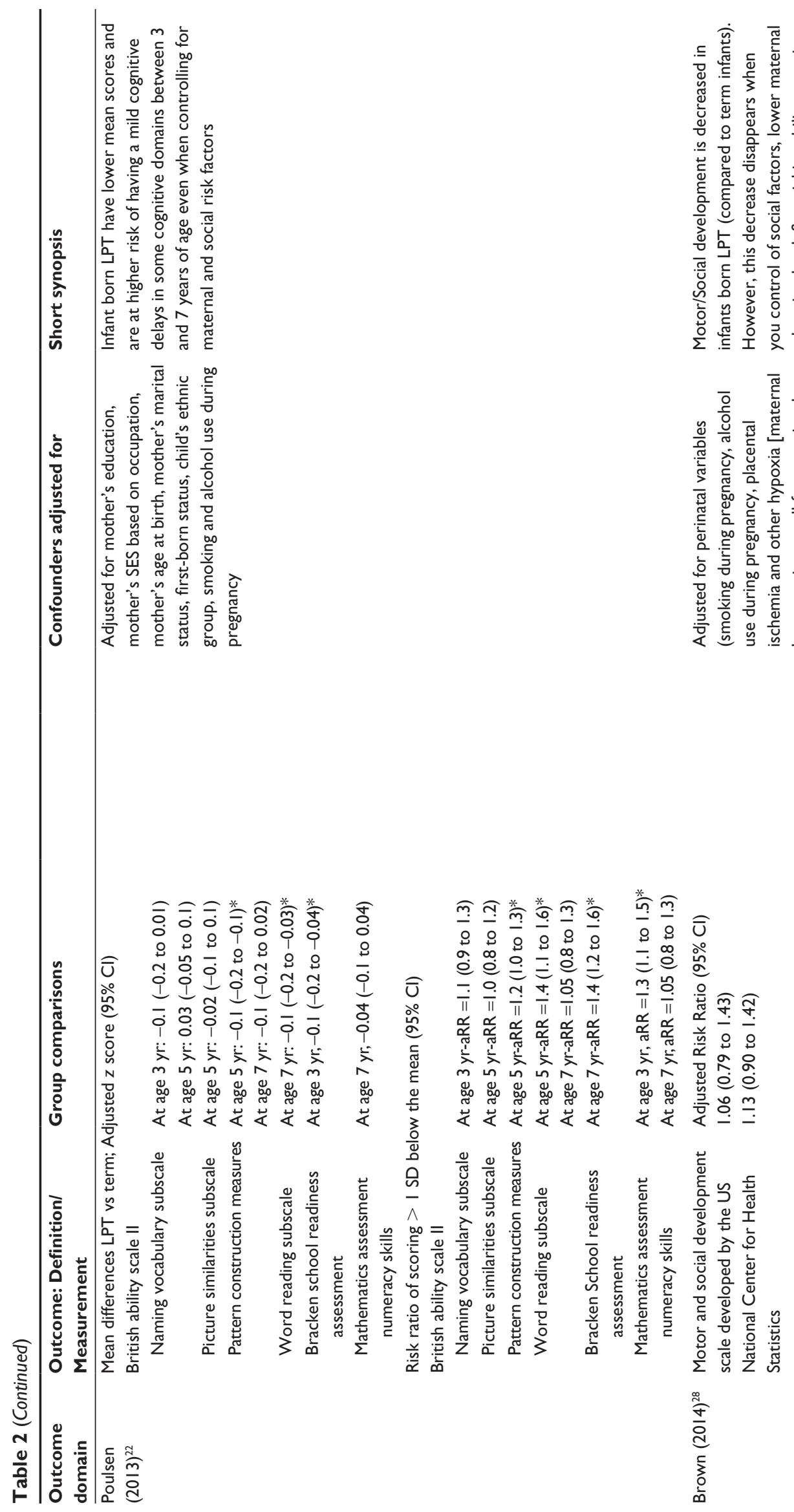



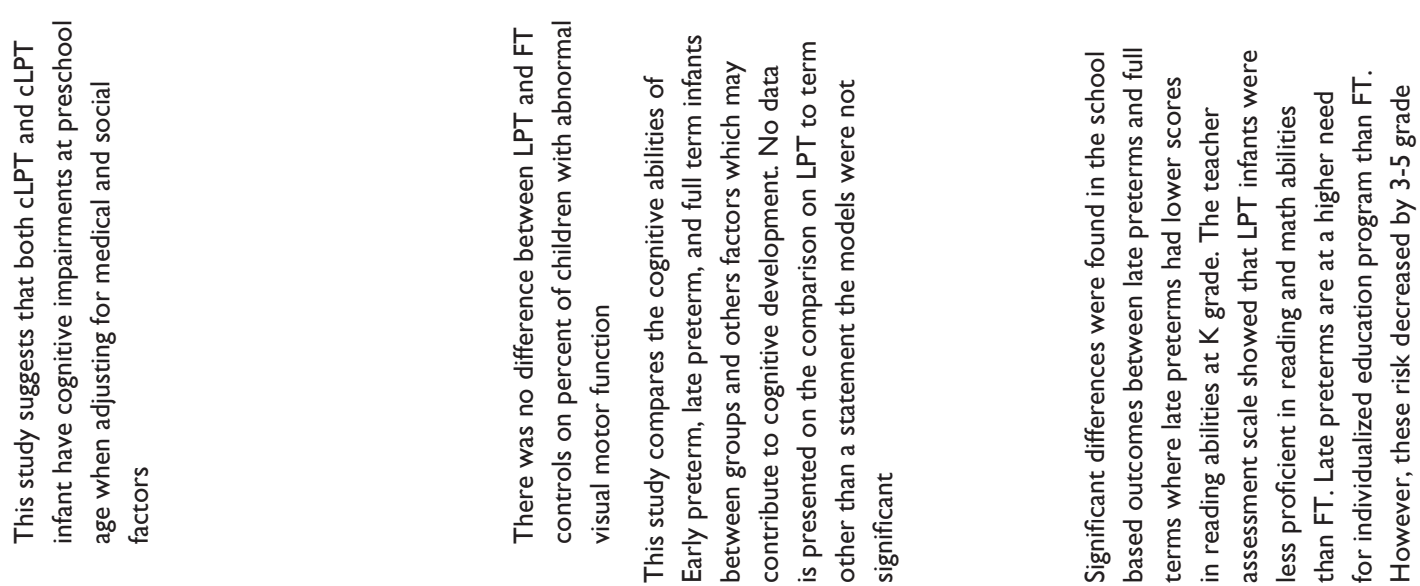

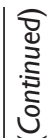
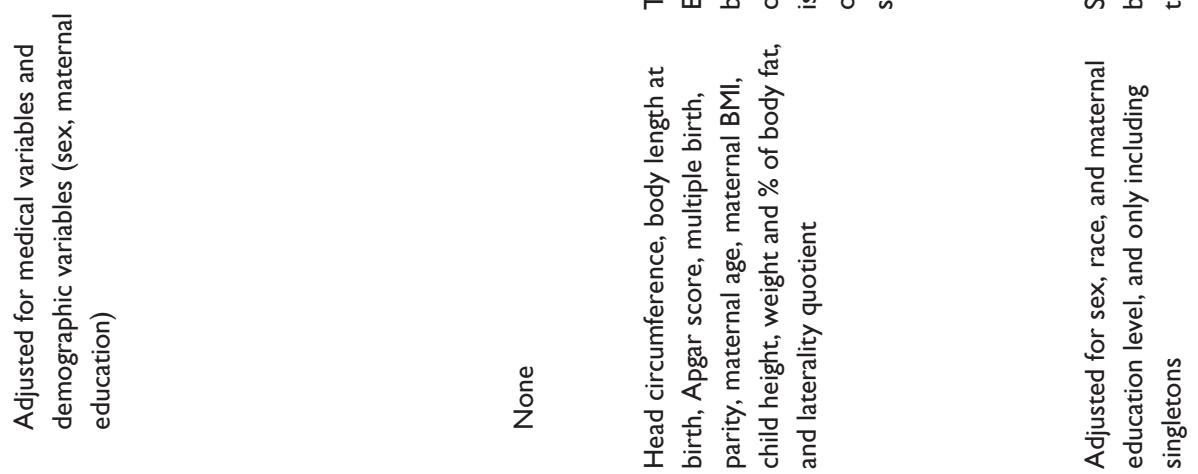

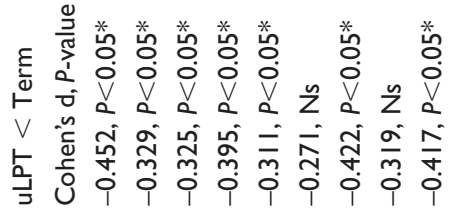
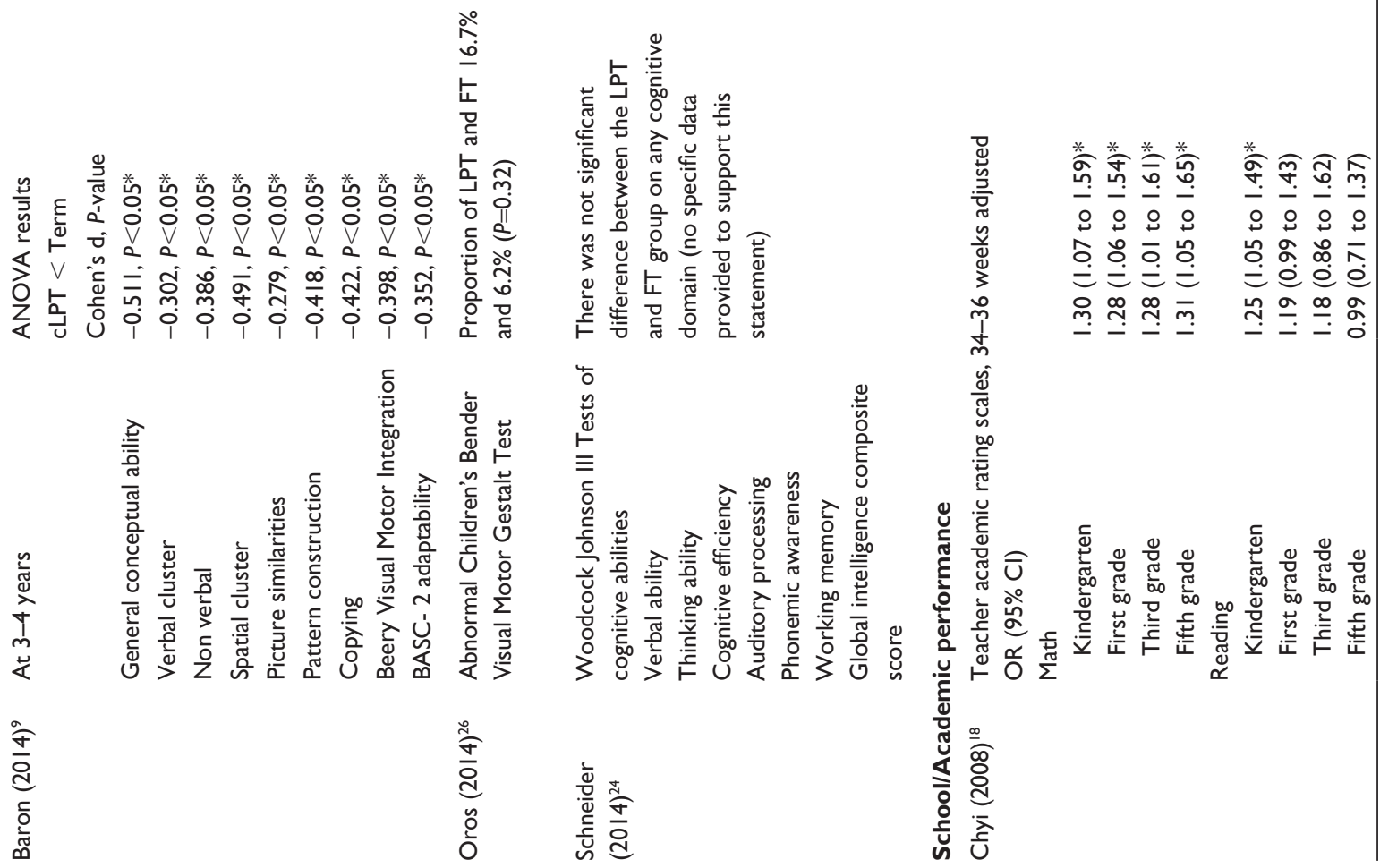


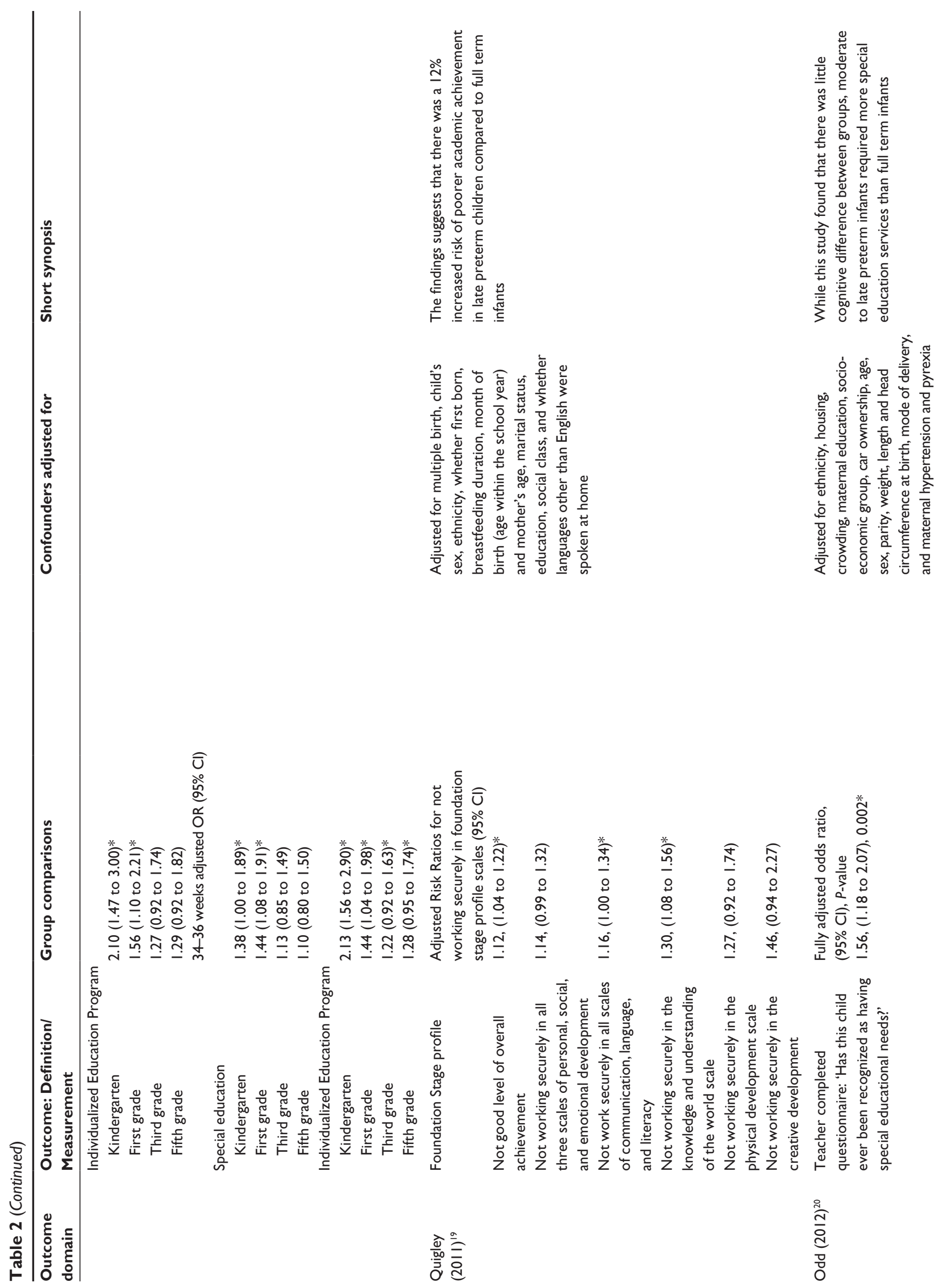



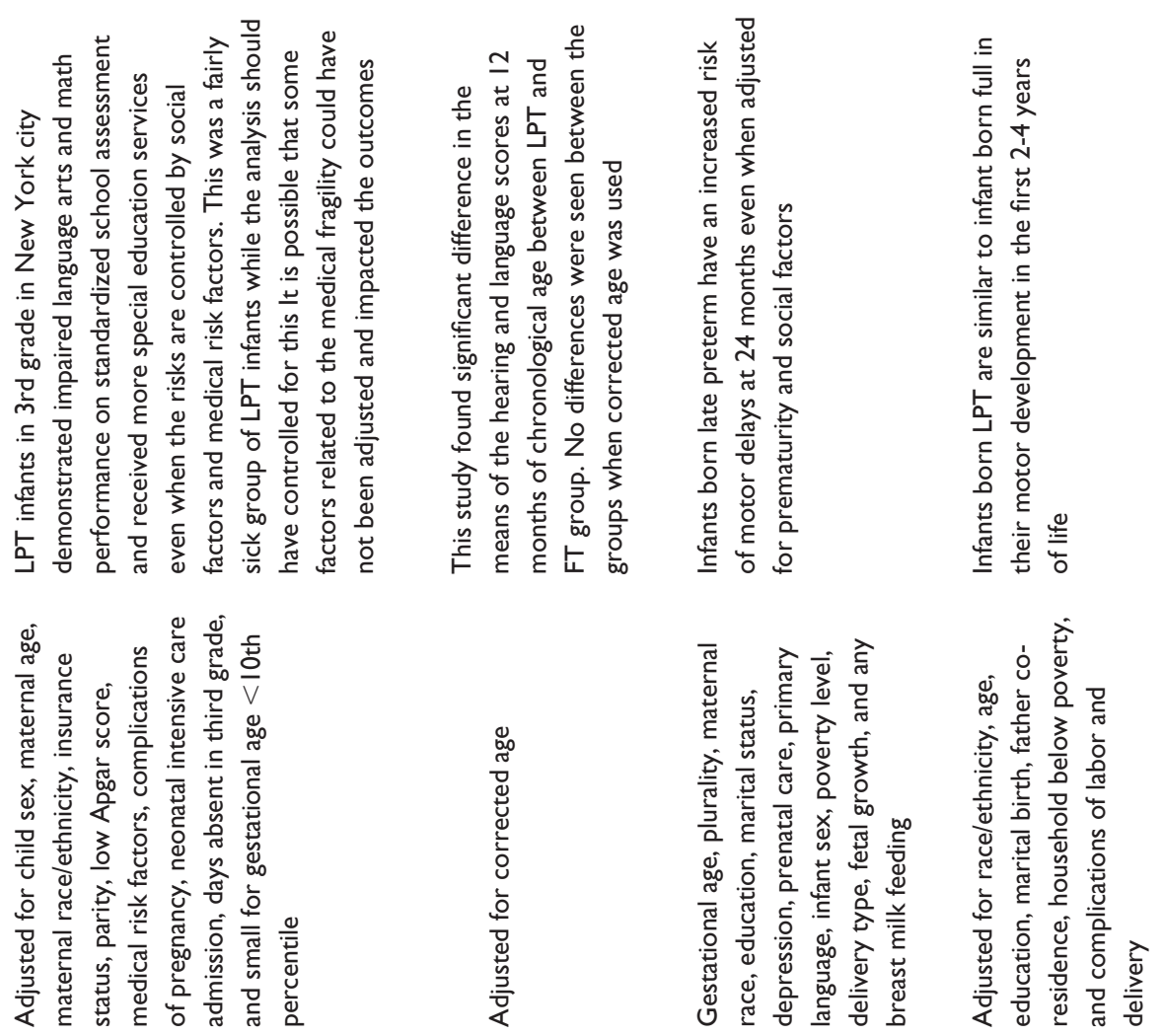

$\frac{0}{\frac{0}{\pi}} \frac{*}{8}$

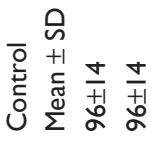

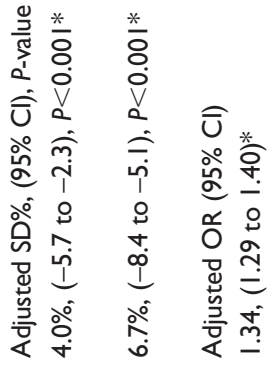
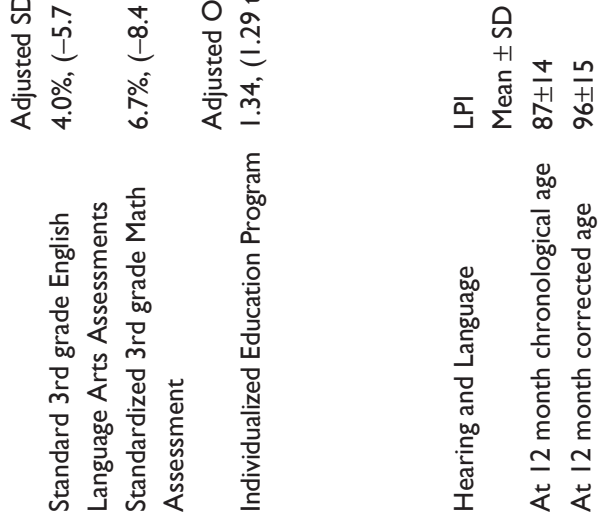

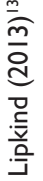
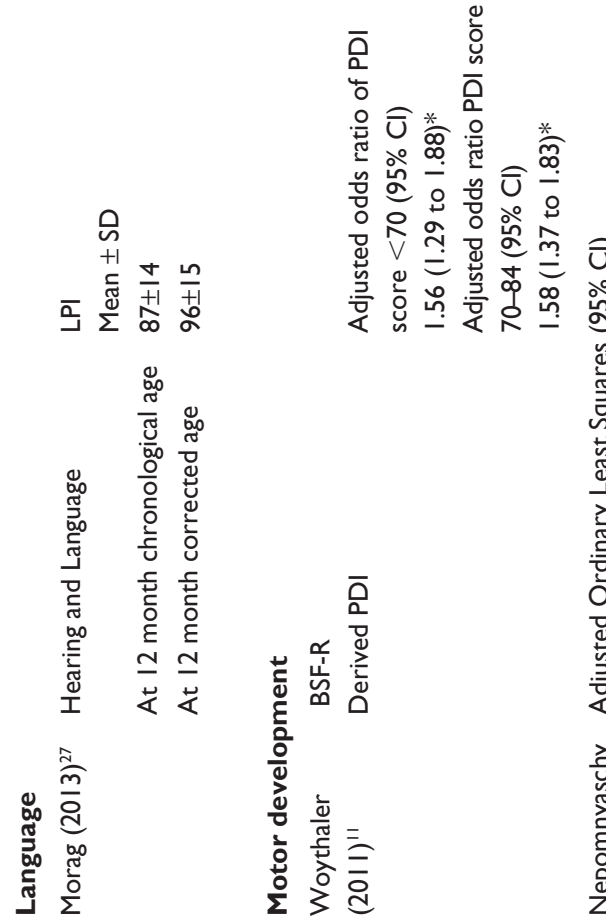

z $\frac{n}{z}$ ž

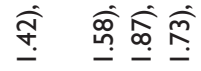

$\therefore \quad 8 \%$

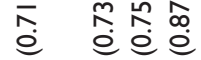

\&. $\quad$ ơ

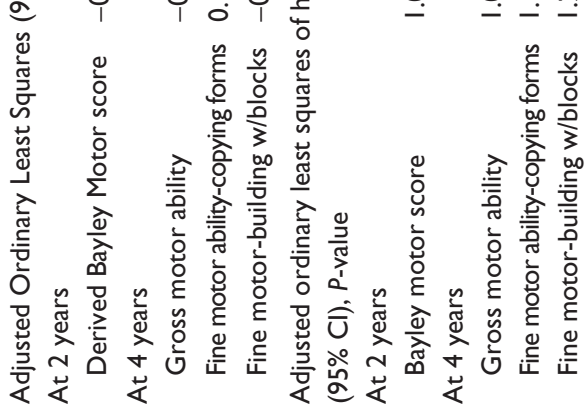

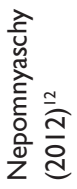




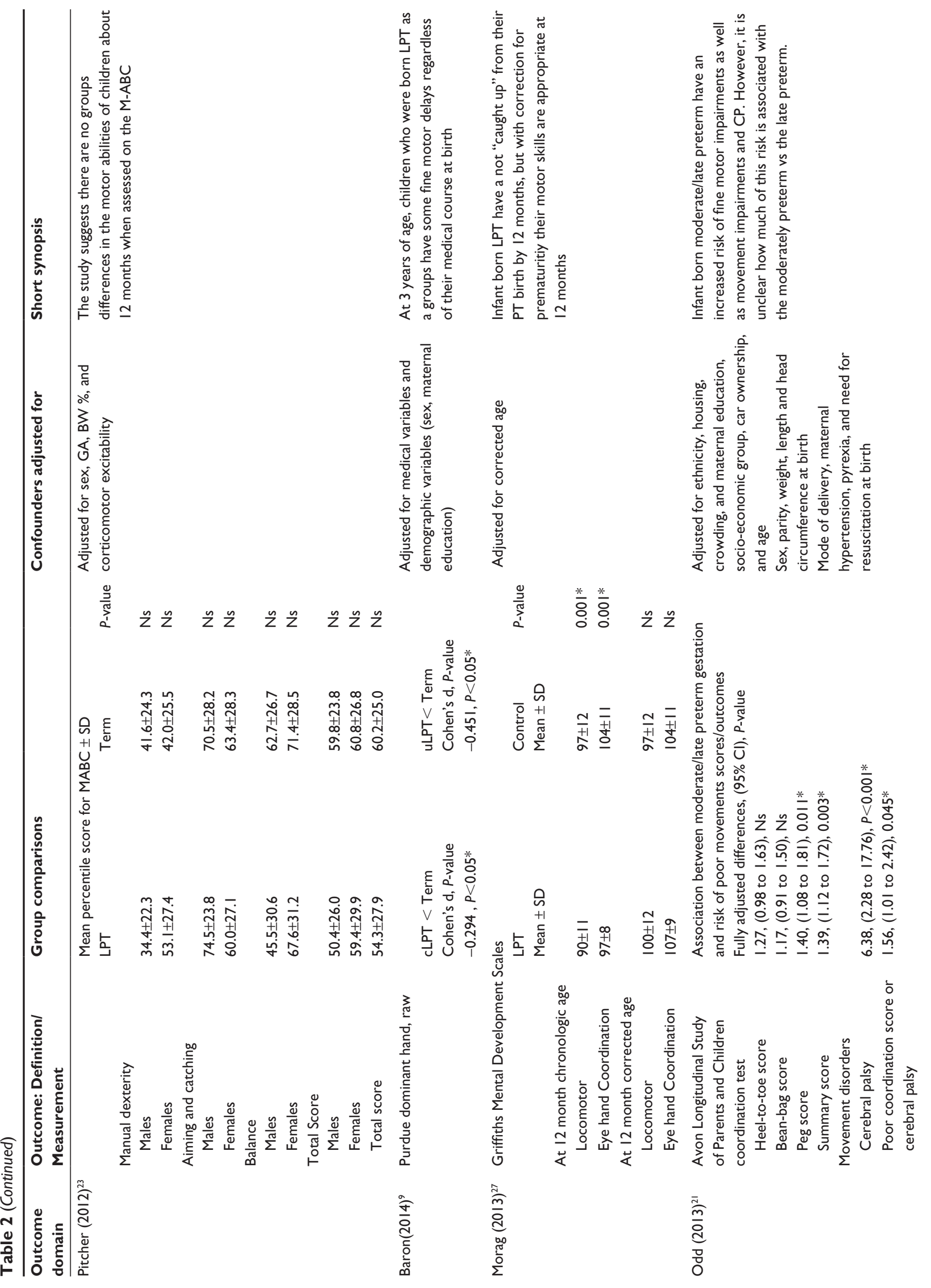



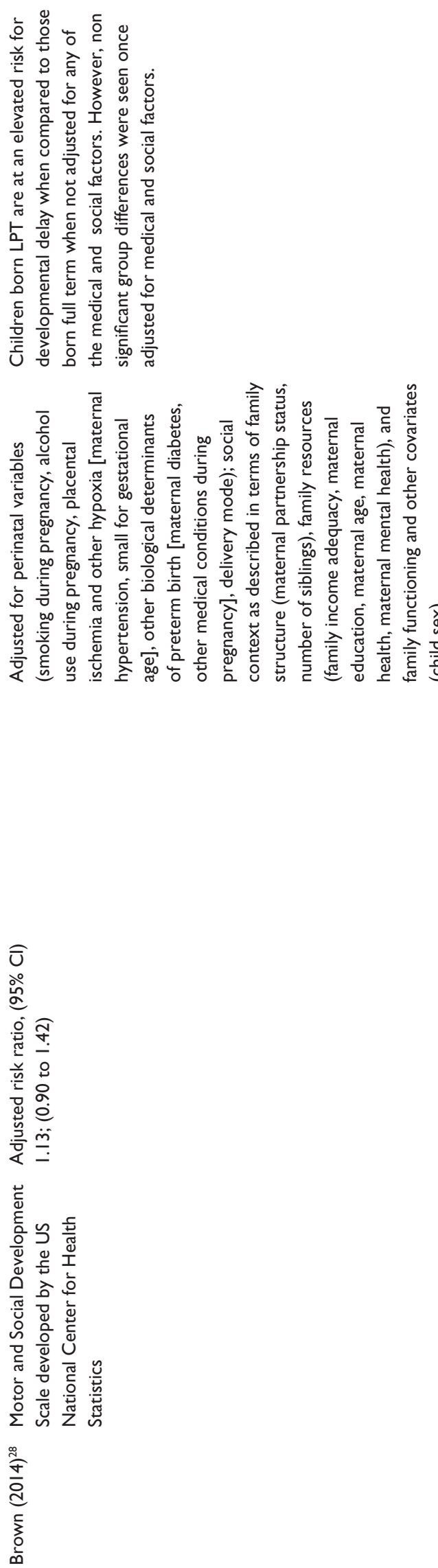

\section{Cognitive studies}

Sixteen studies analyzed for the current review included cognitive development as one of the outcome measures..$^{9-12,14-18,20,22,24-28}$ Under cognition, the subdomains commonly measured are: general conceptual ability, executive function, vocabulary, verbal ability, attention, and memory. The scales commonly used are the Bayley scales of infant development, second edition (Bayley-II), the differential ability scales, second edition (DAS-II), the Griffiths mental development scales (GMDS), and the WoodcockJohnson III test of cognitive abilities. The Wechsler intelligence scale for children and British ability scale are also commonly used.

Seventy-five percent of the 16 studies that assessed cognitive outcomes suggested that as a group, infants born LPT are at increased risk of having cognitive scores that are lower than full term infants, or lower than the average range in at least one of the cognitive domains assessed.

Eight of the 16 studies assessed at least one of the following cognitive abilities: verbal ability, vocabulary, language use, receptive and expressive language, and literacy at ages ranging from 2 to 13 years. ${ }^{9,12,14,15,17,20,22,24}$ Significant differences between LPT and full term groups were seen in language use at age 2 years, ${ }^{12}$ and significant differences were also seen in the domains of literacy and receptive language at age 4 years. ${ }^{12}$ Similarly, significant differences were seen in verbal cluster ability at ages 3-4 years when complicated and uncomplicated LPTs were compared with their full term counterparts. ${ }^{9}$ At ages $6-11$ years, no differences were seen in verbal ability. ${ }^{15,24}$

Four of the 16 studies measured reading and mathematical abilities at ages ranging from 3 to 11 years, and researchers found LPT groups had lower scores in at least one domain at ages 3-6 years when compared to their full term counterparts. ${ }^{12,18,20,22}$ Only one study found significant difference in the reading at $>7$ years of age. ${ }^{20}$ The risk of having a mathematics score more than one standard deviation below sample means was not significant at 4 years of age. ${ }^{12}$

Four of the 16 studies measured intelligence quotient (IQ) or mental abilities by using the Bayley and Wechsler intelligence scales for children. ${ }^{11,12,15,20}$ Two studies suggested significant differences in performance IQ scores at 6-7 years of age $(P<0.05)$ and a higher odds of having a lower Mental Developmental Index (MDI) score and poor IQ when compared to their full term counterparts at 24 months of chronological age and 6-7 years of age, respectively. ${ }^{11,15}$

Two of the 16 studies measured visuomotor function in complicated LPT infants. ${ }^{9,17}$ The studies found significant 


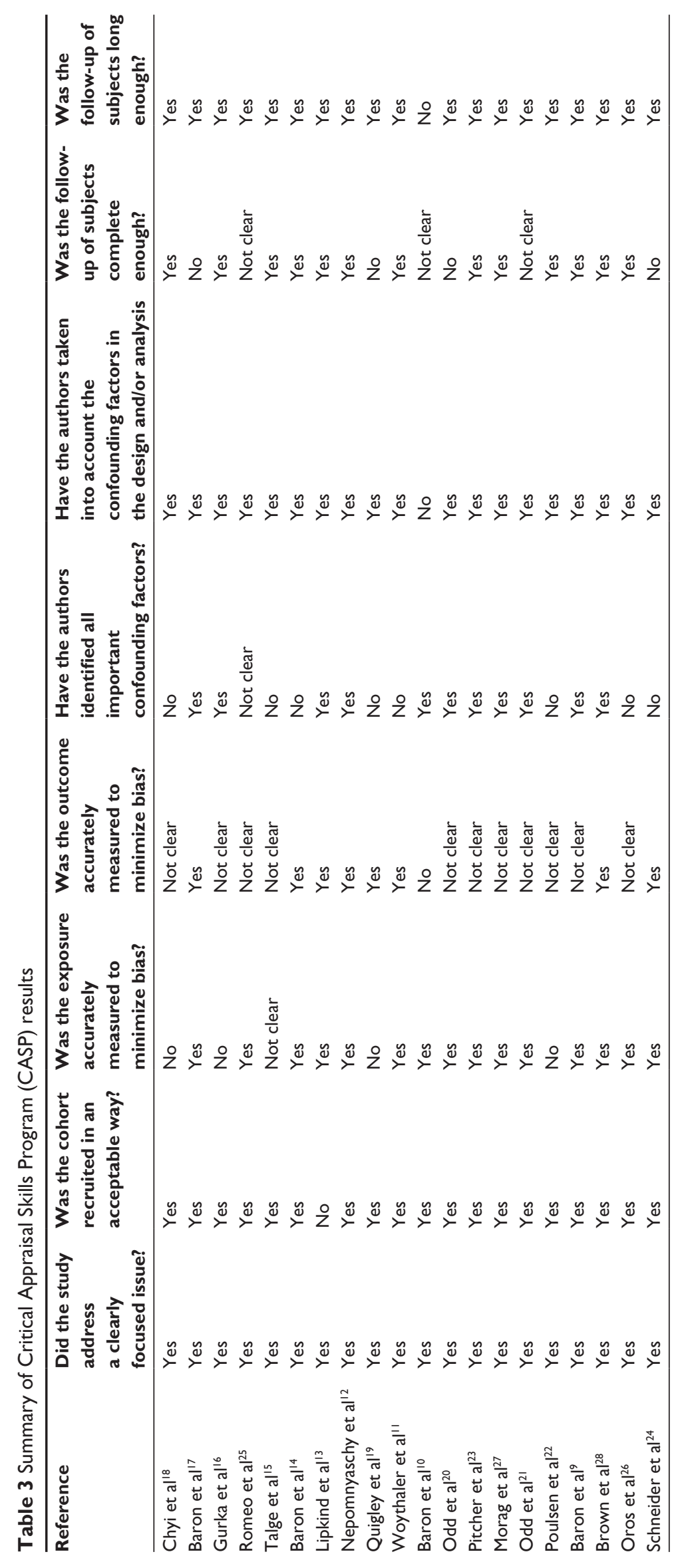


differences in the visuomotor score of LPT infants when compared to those born full term at 3-4 years of age. The differences persisted in both studies even after adjusting for medical and demographic variables..$^{9,17}$

Five studies measured spatial cluster, pattern construction, picture completion, and picture similarities as one of their outcome measures, of which four studies found significant differences between the LPT group and full term group when the groups were assessed at 3-5 years of age., ${ }^{9,14,17,20,22}$ Three of these studies compared the full terms against complicated LPTs and found significant differences at less than 7 years of age. ${ }^{9,14,17}$ Only one ${ }^{22}$ of the two studies ${ }^{20,22}$ that compared a broad group of LPT and full term infants on these domains found a significant result.

Four of the 16 studies did not find statistically significant results in any of the cognitive measures, suggesting no difference between the LPT and full term group. ${ }^{16,24,26,28}$ The cognitive measures used were the Woodcock-Johnson III tests of cognitive abilities, the Abnormal Children's Bender Visual Motor Gestalt Test, and the motor and social development (MSD) scale. One paper had no details of the analysis results, ${ }^{24}$ one paper had a small sample, which may have reduced the power to find significant differences $;{ }^{16}$ and one paper only compared a proportion of children with visual motor abnormalities, limiting the depth of the cognitive domains assessed. ${ }^{26}$ As described in detail in Table 2, studies often had a mixture of significant and non significant findings in different assessment subtests.

\section{Motor development studies}

Seven studies included measures of motor development as one of their outcomes. ${ }^{911,12,21,23,27,28}$ Gross motor development, fine motor development, and coordination were the outcomes commonly assessed using the GMDS, Bayley-II, Bayley short form, research edition (BSF-R), Movement assessment battery for children (MABC), the ALSPAC coordination test, and the MSD scale developed by the US National Center for Health Statistics.

More than $50 \%$ of the included papers which compared the motor development of LPT and full term infants reported motor delays in the LPT cohort. ${ }^{911,21,27}$ LPT and full term groups differed in gross motor abilities until 3 years of age. Only one study reported a difference in fine motor abilities at the age of 7 years. ${ }^{21}$ However, the LPT and moderately preterm (34-35 weeks of gestation) groups were combined and were analyzed together, which may have increased the likelihood of finding significant differences. ${ }^{21}$ The only study that compared motor development differences of complicated and uncomplicated LPT infants against full terms at 3-4 years of age found a significant difference in manual dexterity between both LPT groups and the full term infants. ${ }^{9}$

\section{School/academic performance studies}

Four studies included school/academic performance as one of their developmental outcomes. ${ }^{13,18-20}$ The subjects' ages at assessment ranged from 5 to 11 years of age. Teachercompleted questionnaires, academic rating scales, and the need for individualized education plans were the measures commonly used.

All four papers identified reduced academic performance or an increased need for special education services in the infants born LPT. Chyi et al ${ }^{18}$ found lower academic ratings in mathematical ability until fifth grade in children born LPT when compared to their full term counterparts. Quigley et a ${ }^{19}$ suggested a $12 \%$ increase in the risk of poorer academic achievement in children born LPT compared to those born at term. Studies assessing the need for special education and individualized education programs have found that children born LPT have an increased need and require more special educational services compared to their full term counterparts. ${ }^{13,18,20}$

\section{Language study}

Only one study compared hearing and language in LPT and full term infants. ${ }^{27}$ The authors reported statistically significant differences $(P=0.001)$ when comparing the full term group with the LPT group at 12 months of chronological age. However, there was no difference when comparing the full term group with the LPT group if the LPT scores were calculated using the infants' adjusted age. ${ }^{27}$

\section{Quality of the papers}

The quality of the methods included in the 20 papers included in the current systematic review was fair overall (Table 3). Some common limitations were observed, including small sample sizes in the studies that followed infants with the most detailed assessments and those with complicated preterm infants. A lack of clarity or precision in measuring gestational age was another common limitation. ${ }^{15,16,18,19,22}$ In 12 studies, it was unclear whether blinding techniques were used, or there was no blinding of the outcome measures. . $^{9} 15,16,18,20-23,25-28$ While confounding factors such as maternal education level, race, and poverty status were often documented and in some cases adjusted for in the analyses, few studies included information on the medical status of the infants in the study. This was particularly true of the largest studies using national datasets, where information on medical status or the length of hospital stay at birth may not have been present. 


\section{Discussion}

The findings of the present systematic review suggest that infants born LPT, as a group, are at an increased risk of having neurodevelopmental outcomes that are worse than infants born full term even, when social and medical risk factors are controlled for. Although the lower outcomes are not present in all domains and appear to improve over time in most areas, the findings are consistent with a previous systemic review. ${ }^{7}$ The most evidence is available on the outcomes of infants born LPT in the cognitive domain. However, for all domains, the age of assessment, use of corrected vs chronological age, and medical fragility must be considered in interpreting the results, in addition to controlling for social factors that are known to increase the rate of developmental delays. In addition, the relationship between cognitive scores and academic performance is of great importance. The following three "Outcomes" sections discuss the results by age group, along with the special considerations that are most relevant to each age group.

\section{Outcomes from I to 2 years of age}

At 24 months of age, there were conflicting outcomes regarding the cognitive and motor outcomes of LPT infants evident in the literature. Woythaler et $\mathrm{al}^{11}$ and Nepomnyaschy et $\mathrm{al}^{12}$ completed their research using the same database, the ECLS-B, yet they found conflicting results. This disparity could be explained by the differences in their interpretation of the data and their use of statistical analyses. Woythaler et $\mathrm{al}^{11}$ compared their data using standardized scores and found significant group differences in cognitive abilities at 24 months. In contrast, Nepomnyaschy et al ${ }^{12}$ converted the cognitive and motor scores to percentiles prior to analysis, which may have reduced the ability to detect group differences due to the limitations of using percentiles in statistical analysis.

While it is common practice to use corrected or adjusted age (chronological age minus weeks' preterm) during developmental assessments on an extremely premature infants, this practice is less commonly used with infants born LPT. In five papers, four cognitive outcome measures ${ }^{11,12,25,27}$ and one language outcome measure ${ }^{27}$ were assessed in infants at $<2$ years of age, and outcomes showed significant group differences when scored using the infants' chronological age, rather than adjusting the age for prematurity. However, when each infant's corrected age was used to score the outcome measures, the groups appeared similar in their outcome measures. ${ }^{25,27}$ This comparison suggests that LPT infants are not "catching up" developmentally with those born at full term by $1-1.5$ years of age. No study included in the current review used a corrected age at or after 2 years of age, or compared the scores on corrected vs chronological age, limiting our ability to identify when and if the infants born LPT "catch up" with their full term peers. In addition, clinical differences in the use of corrected ages may alter if a child is identified as having a delay. Based on the papers included in the present systematic review, children at $1-1.5$ years of age have lower cognitive and language scores when scored using a chronological age than infants born full term. There were no papers that assessed motor outcomes or school readiness between 1 and 2 years of age.

\section{Outcomes from 3 to 5 years of age}

At 3-5 years of age, cognitive, motor, and language assessments are more detailed, and assessments are divided between more conceptual areas. While the wide variety of assessments and lack of consistency on the conceptual areas measured makes interpretation challenging, deficits in the cognitive areas of literacy, general conceptual ability, vocabulary, and mathematics are noted in this age range. Brown et $\mathrm{al}^{28}$ and Nepomnyaschy et $\mathrm{al}^{12}$ assessed cognition at 3-5 years of age. Their findings were inconsistent, as Nepomnyaschy et al ${ }^{12}$ found significant group differences in vocabulary, literacy, receptive language, and mathematical ability, while Brown et $\mathrm{al}^{28}$ found no group differences. The inconsistency can be explained by the difference in the cognitive measures used in the outcome assessment. Nepomnyaschy et al ${ }^{12}$ focused on more detailed conceptual constructs of cognition; Brown et $\mathrm{al}^{28}$ used a general measure, ie, the MSD scale. This suggests that with a general measure, the infants born LPT and full term may score similarly, possibly because scores on some items inflate the overall score, thus reducing the likelihood of detecting more subtle deficits in conceptual areas. In contrast, the more detailed assessment of specific conceptual constructs allows the examiner to determine an understanding of strengths and weaknesses within the broader cognitive domain.

No significant group differences were seen between the LPT and full term groups in the motor domain at 3-5 years of age. Several studies $9,10,12,14,16,17,22,28$ compared the motor and cognitive outcomes of 3-5-year-old children born LPT, with and without medical complications. The findings of these studies suggest that infants with medical complications requiring a prolonged hospitalization or stay in a neonatal intensive care unit are at higher risk than infants born LPT who did not have medical complications. However, three 
studies that compared medically complicated and uncomplicated LPT infants were completed in one medical center, limiting the generalizability of these results. In addition, there are no studies of the outcomes of these infants at school age or older.

\section{Outcomes from 6 years of age and older}

The findings of the included studies assessing cognition in children 6 years of age and older identified poor performance, lower IQ, and lower scores in reading and mathematics when compared with the full term group..$^{15,18,22}$ It is noteworthy that group differences are attenuated and become less or even nonsignificant with an increase in the age of assessment, ie, assessment performed at $11-15$ years of age. ${ }^{16,18,20}$ In addition, in multiple studies, there is a difference in the cognitive scores between groups; however, the scores of both groups are in the average range for the assessment utilized. ${ }^{25,27}$ This result suggests that as a group, LPT infants have lower cognitive function in later years, but possibly not to the level of cognitive impairment in the early school years.

While many studies found that cognitive scores were only different on a few measures by school age, the number of children who were under-performing in school or who required special education was different between groups. ${ }^{13,18,20}$ This fact suggests that while the infants born LPT may only have small gaps in their cognitive capacity, the gaps, combined with the other issues such as sensory processing or attention (which were not reviewed in this paper), may be resulting in reduced academic performance and a greater need for special education support in the school.

The present systematic review has some limitations that may have influenced the identification of papers or interpretation of our results. First, our key search terms restricted our search to four domains of neurodevelopment: the cognitive, motor, language, and school/academic performance domains. Thus, we did not include studies discussing social, emotional, or behavioral outcomes, or the diagnosis of specific conditions. Thus, we cannot comment on the presence of other impairments in the populations or their impact on the neurodevelopmental outcomes we assessed. Second, we focused on the outcomes, which in most cases had been statistically adjusted for social and medical factors. Thus, the results are likely a significant under-representation of the neurodevelopmental impairments in the entire population of children born LPT. This confounding factor may also account for some of the differences in cognitive and school outcomes. Infants born LPT who have mild cognitive impairment, but have access to highly qualified teachers, tutors, and educated parents may be able to compensate for their impairments, while children without these resources are less successful in school. Additional analysis of the outcomes, using both adjusted and non adjusted values, is required to begin to address this issue. Lastly, we have only discussed the design aspect of the included studies, and we did not weigh analysis quality of the included studies in our interpretation of results.

Further research is needed to fill in some gaps in the literature and to expand our understanding of the developmental outcomes of infants born LPT. Researchers aiming to work on the neurodevelopment of LPT infants should include a control/ full term group in their study design along with confounding neonatal medical variables in order to determine post-birth fragility status. The inclusion of a healthy and a medically complex group of LPT infants would also add considerable value to all future studies. It is essential to note that recruiting the LPT group based on parental reports of gestational age raises questions about the accuracy of the exposure measured. A consistent use of outcome measures between studies would also greatly increase comparison power between future studies. Lastly, researchers and clinicians need to consider the chronological age and corrected age of infants born LPT until at least 2 years of age, and furthermore, they should document outcomes for both chronological and corrected ages until we have a better understanding of when and if infants born LPT do catch up to their peers.

\section{Conclusion}

In conclusion, infants born LPT are at a risk of having reduced long-term neurodevelopmental outcomes, with cognition being at the highest risk and persisting the longest. Physicians, early intervention, and early childhood education programs, policies makers, parents, therapists, and teachers need to be aware of the long-term neurodevelopmental risks associated with LPT birth. While the risks maybe be lower for LPT births than for extremely preterm infants, systematic follow-up and/or assessment at school entry be beneficial to improve the outcomes of infants born LPT.

\section{Acknowledgment}

The authors would like to thank Ms Gullnar Syed, psychology intern at Motor Development Lab, Virginia Commonwealth University (VCU) and Richmond, VA, USA for helping them to locate and review papers for the current systematic review.

\section{Disclosure}

The authors report no conflicts of interest in this work. 


\section{References}

1. Raju TNK, Higgins RD, Stark AR, Leveno KJ. Optimizing care and outcome for late-preterm (near-term) infants: a summary of the workshop sponsored by the National Institute of Child Health and Human Development. Pediatrics. 2006;118(3):1207-1214.

2. Engle WA, Tomashek KM, Wallman C. "Late-preterm" infants: a population at risk. Pediatrics. 2007;120(6):1390-1401.

3. Baron IS, Litman FR, Ahronovich MD, Baker R. Late preterm birth: a review of medical and neuropsychological childhood outcomes. Neuropsychology review. 2012;22(4):438-450.

4. Loftin RW, Habli M, Snyder CC, Cormier CM, Lewis DF, Defranco EA. Late preterm birth. Reviews in obstetrics and gynecology. 2010;3(1):10-19.

5. Shapiro-Mendoza C, Tomashek KM, Kotelchuck M, Barfield W, Weiss J, Evans S. Risk factors for neonatal morbidity and mortality among "healthy," late preterm newborns. Seminars in perinatology. 2006;30(2):54-60.

6. Melamed N, Klinger G, Tenenbaum-gavish K, et al. Short-term Neonatal Outcome in Low-Risk, Spontaneous, Singleton, Late Preterm Deliveries. Obstetrics and Gynecology. 2009;114(2):253-260.

7. McGowan J, E., Alderdice F, A., Holmes V, A., Johnston L. Early Childhood Development of Late-Preterm Infants: A Systematic Review. Pediatrics. 2011;127(6):1111-1124. doi: 10.1542/peds.2010-2257.

8. Critical Appraisal Skills Programme [webpage on the Internet]. Oxford: Critical Appraisal Skills Programme. Available from: http://media.wix. com/ugd/dded87_e37a4ab637fe46a0869f9f977dacf134.pdf. Accessed January, 2015.

9. Baron IS, Weiss BA, Baker R, et al. Subtle adverse effects of late preterm birth: a cautionary note. Neuropsychology. 2014;28(1):11-18.

10. Baron IS, Kerns KA, Müller U, Ahronovich MD, Litman FR. Executive functions in extremely low birth weight and late-preterm preschoolers: effects on working memory and response inhibition. Child neuropsychology. 2012;18(6):586-599.

11. Woythaler MA, McCormick MC, Smith VC. Late preterm infants have worse 24-month neurodevelopmental outcomes than term infants. Pediatrics. 2011;127(3):e622-e629.

12. Nepomnyaschy L, Hegyi T, Ostfeld BM, Reichman NE. Developmental outcomes of late-preterm infants at 2 and 4 years. Maternal and child health journal. 2012;16(8):1612-1624.

13. Lipkind HS, Slopen ME, Pfeiffer MR, McVeigh KH. School-age outcomes of late preterm infants in New York City. AM J OBSTET GYNECOL. 2012;206(3):222.e1-6.

14. Baron IS, Erickson K, Ahronovich MD, Baker R, Litman FR. Cognitive deficit in preschoolers born late-preterm. Early Hum Dev. 2011;87(2):115-119. doi: 10.1016/j.earlhumdev.2010.11.010.
15. Talge NM, Holzman C, Wang J, Lucia V, Gardiner J, Breslau N. Latepreterm birth and its association with cognitive and socioemotional outcomes at 6 years of age. Pediatrics. 2010;126(6):1124-1131.

16. Gurka MJ, LoCasale-Crouch J, Blackman JA. Long-term cognition, achievement, socioemotional, and behavioral development of healthy late-preterm infants. Archives of pediatrics and adolescent medicine. 2010;164(6):525-532.

17. Baron IS, Erickson K, Ahronovich MD, Coulehan K, Baker R, Litman FR. Visuospatial and verbal fluency relative deficits in 'complicated' late-preterm preschool children. Early Hum Dev. 2009;85(12):751-754. doi: 10.1016/j.earlhumdev.2009.10.002.

18. Chyi LJ, Lee HC, Hintz SR, Gould JB, Sutcliffe TL. School outcomes of late preterm infants: special needs and challenges for infants born at 32 to 36 weeks gestation. The journal of pediatrics. 2008;153(1):25-31.

19. Quigley MA, Poulsen G, Boyle E, et al. Early term and late preterm birth are associated with poorer school performance at age 5 years: a cohort study. Archives of disease in childhood. 2012;97(3):F167-F173.

20. Odd DE, Emond A, Whitelaw A. Long-term cognitive outcomes of infants born moderately and late preterm. Developmental medicine and child neurology. 2012;54(8):704-709.

21. Odd DE, Lingam R, Emond A, Whitelaw A. Movement outcomes of infants born moderate and late preterm. Acta padiatrica. 2013;102(9):876-882.

22. Poulsen G, Wolke D, Kurinczuk JJ, et al. Gestational age and cognitive ability in early childhood: a population-based cohort study. Paediatric and perinatal epidemiology. 2013;27(4):371-379.

23. Pitcher JB, Schneider LA, Burns NR, et al. Reduced corticomotor excitability and motor skills development in children born preterm. The journal of physiology. 2012;590:5827-5844.

24. Schneider LA, Burns NR, Giles LC, et al. Cognitive abilities in preterm and term-born adolescents. The journal of pediatrics. 2014;165(1):170-177.

25. Romeo DM, Di Stefano A, Conversano M, et al. Neurodevelopmental outcome at 12 and 18 months in late preterm infants. European journal of paediatric neurology. 2010;14(6):503-507.

26. Oros D, Altermir I, Elia N, et al. Pathways of neuronal and cognitive development in children born small-for-gestational age or late preterm. Ultrasound in obstetrics and gynecology. 2014;43(1):41-47.

27. Morag I, Bart O, Raz R, et al. Developmental characteristics of late preterm infants at six and twelve months: a prospective study. Infant behavior and development. 2013;36(3):451-456.

28. Brown HK, Speechley KN, Macnab J, Natale R, Campbell MK. Mild prematurity, proximal social processes, and development. Pediatrics. 2014;134(3):e814-e824. 


\section{Supplementary materials \\ Search terms \\ PubMed database}

Keywords: ((("late preterm infants") OR ((“Premature Birth"[Mesh]) OR "Infant, Premature"[Mesh]))) AND ((“Child Development”[Mesh]) OR “developmental outcomes" OR "Motor" OR "Cognitive" OR "Language" OR "School performance" OR "Skill”).

(("Child Development”[Mesh] OR "motor development" OR "cognitive development" OR "language development" OR "school performance" OR "developmental outcomes")) AND (("Infant, Premature"[Mesh]) OR "Premature Birth"[Mesh] OR ("late preterm infants") OR ("premature infants")).

\section{CINAHL database}

Search terms: (MM "Child Development") OR (MH "Infant Development") OR (MH "Language Development") OR (MH "Child Development: 12 Months (Iowa NOC)") OR (MH "Child Development: 2 Years (Iowa NOC)") OR (MH "Child Development: 3 Years (Iowa NOC)") OR (MH "Child Development: 4 Years (Iowa NOC)") OR (MH
"Child Development: 5 Years (Iowa NOC)") OR (MH "Child Development: Middle Childhood (6-11 Years) (Iowa NOC)") OR (MH "Child Development: Adolescence (12-17 Years) (Iowa NOC)") OR (MH "Cognition") OR (MH "Perception") OR "Cognitive development" OR (MH "Psychomotor Performance") OR (MH "Learning”) OR (MH "Mental Processes") OR "Motor development” OR (MH “Academic Performance") And (MH “Infant, Premature").

\section{PsycINFO database}

\{Cognitive Development\} OR \{Fine Motor Skill Learning OR \{Gross Motor Skill Learning\} OR \{Infant Development\} OR \{Language Development\} OR \{Motor Development\} OR \{Motor Performance\} OR \{Motor Skills\} OR \{Perceptual Motor Learning\} OR \{Skill Learning $\}$ And Premature Infant.

\section{ERIC database}

(Child development OR Motor development OR Cognitive development OR Language development OR School performance OR Skill AND late preterm OR preterm infant OR premature infant OR premature birth) AND (late preterm OR premature birth) OR (preterm infant OR premature infant).

\section{Publish your work in this journal}

Research and Reports in Neonatology is an international, peer-reviewed, open access journal publishing original research, reports, editorials, reviews and commentaries on neonatal health. The manuscript manage-

peer-review system. Visit http://www.dovepress.com/testimonials.php ment system is completely online and includes a very quick and fair 